\title{
A Short Proof for the Krull Dimension of a Polynomial Ring
}

\author{
Thierry Coquand and Henri Lombardi
}

A first important result in dimension theory is the fact that the Krull dimension of the ring $K\left[X_{1}, \ldots, X_{\ell}\right]$ is equal to $\ell$ when $K$ is a field. In the literature this result is always obtained after some preliminary efforts that seem excessive for settling such an intuitive fact. For example, many authors rely on the principal ideal theorem of Krull, whose proof is very tricky. Matsumura [6,chap.2] gives a rather direct proof, but his Theorem 5.6 settling the result needs Theorems 5.1 to 5.5 together with three pages of rather subtle arguments, including Hilbert's Nullstellensatz. The shortest proof we are aware of appears in [3]. The goal of this note is to give a short, direct proof of this fact, based on an elementary elementwise characterization of Krull dimension (Corollary 2).

Let $R$ be a nontrivial commutative ring. Recall that the Krull dimension of $R$ is the maximal length $\ell$ of a chain $\mathfrak{p}_{0} \subset \mathfrak{p}_{1} \subset \cdots \subset \mathfrak{p}_{\ell}$ of prime ideals inside $R$. By convention, a ring $R$ has Krull dimension -1 if and only if it is trivial (i.e., $1_{R}=0_{R}$ ). Since the notion of Krull dimension is very important in commutative algebra (see, for example, part II of Eisenbud [3]), it is useful to give a purely elementary characterization of Krull dimension, without invoking prime ideals.

We define the boundary $R_{\{x\}}$ of $x$ in $R$ as the localized ring $S_{\{x\}}{ }^{-1} R$, where

$$
S_{\{x\}}=x^{\mathbb{N}}(1+x R)=\left\{z \in R \mid \exists n \in \mathbb{N}, \exists a \in R, z=x^{n}(1+a x)\right\} .
$$

Note that $R_{\{x\}}$ is trivial if $x$ is invertible or nilpotent; passing from $R$ to the boundary $R_{\{x\}}$ is an efficient way to discard invertible and nilpotent elements. This is similar to the consideration of the boundary of a subvariety $W$ in a variety $V$ : the boundary is empty whenever $W$ is empty (corresponding to the equation $1=0$-i.e., $x=0$, with $x$ invertible) or "full" (corresponding to the equation $0=0$-i.e., $x=0$, with $x$ nilpotent).

More precisely we get the following theorem (see [2]):

Theorem 1 Let $R$ be a commutative ring, and let $\ell$ be a nonnegative integer. The following statements are equivalent:

1 The Krull dimension of $R$ is at most $\ell$.

2 For each $x$ in $R$ the Krull dimension of $R_{\{x\}}$ is at most $\ell-1$.

Proof. First recall that a prime ideal of a localization $S^{-1} R$ has the form $S^{-1} \mathfrak{p}$, where $\mathfrak{p}$ is a prime ideal of $R$ not intersecting $S$. We make two claims:

(a) For each $x$ in $R$, every maximal ideal $\mathfrak{m}$ of $R$ intersects $S_{\{x\}}$. Indeed, if $x$ belongs to $\mathfrak{m}$, this is obvious; otherwise $x$ is invertible modulo $\mathfrak{m}$ which implies that $1+x R$ intersects $\mathfrak{m}$.

(b) If $\mathfrak{m}$ is a maximal ideal of $R$ and $x$ belongs to $\mathfrak{m} \backslash \mathfrak{p}$, where $\mathfrak{p}$ is a prime ideal contained in $\mathfrak{m}$, then $\mathfrak{p} \cap S_{\{x\}}=\emptyset$. Namely, if $x^{k}(1+x y) \in \mathfrak{p}$, then since $x \notin \mathfrak{p}$ we get $1+x y \in \mathfrak{p} \subset \mathfrak{m}$, so $1 \in \mathfrak{m}$, contradicting the maximality of $\mathfrak{m}$.

Now the equivalence is easy. Claim (a) shows that any chain of primes ending with a maximal ideal in $R$ is shortened when passing to $R_{\{x\}}$, while it follows from claim (b) that a chain of maximal length $\ell$ is shortened to $\ell-1$ if $x$ is properly chosen.

Starting from dimension -1 , Theorem 1 gives an elementary inductive characterization of Krull dimension. This can be made more explicit through the following corollary (see [1],[5]): 
Corollary 2 Let $\ell$ be a nonnegative integer. The Krull dimension of $R$ is at most $\ell$ if and only if for any given $x_{0}, \ldots, x_{\ell}$ in $R$ there exist $a_{0}, \ldots, a_{\ell}$ in $R$ and $m_{0}, \ldots, m_{\ell}$ in $\mathbb{N}$ such that

$$
x_{0}^{m_{0}}\left(\cdots\left(x_{\ell}^{m_{\ell}}\left(1+a_{\ell} x_{\ell}\right)+\cdots\right)+a_{0} x_{0}\right)=0 .
$$

Proof. For $\ell=0$, this is a exactly Theorem 1. Assume that the equivalence has been established for all integers less than $\ell$ and all $R$. We deduce that the dimension of a localization $S^{-1} R$ is smaller than $\ell$ if and only if for all $x_{0}, \ldots, x_{\ell-1}$ in $R$ there exist $a_{0}, \ldots, a_{\ell-1}$ in $R, s$ in $S$, and $m_{0}, \ldots, m_{\ell-1}$ in $\mathbb{N}$ such that

$$
x_{0}^{m_{0}}\left(x_{1}^{m_{1}} \cdots\left(x_{\ell-1}^{m_{\ell-1}}\left(s+a_{\ell-1} x_{\ell-1}\right)+\cdots+a_{1} x_{1}\right)+a_{0} x_{0}\right)=0 .
$$

Observe that $s$ replaces 1 in the similar equality (1) for $R$ rather than $S^{-1} R$. It remains only to replace $s$ with an arbitrary element in $S_{\left\{x_{\ell}\right\}}$ (i.e., with an element $x_{\ell}^{m_{\ell}}\left(1+a_{\ell} x_{\ell}\right)$ ).

We emphasize the fact that the characterization given in Corollary 2 does not use the set of prime ideals.

Corollary 3 Let $K$ be a field, and let $R$ be a commutative $K$-algebra. If any sequence $x_{0}, \ldots, x_{\ell}$ in $R$ is algebraically dependent over $K$, then the Krull dimension of $R$ is at most $\ell$.

Proof. Let $Q\left(x_{0}, \ldots, x_{\ell}\right)=0$ be an algebraic dependence relation over $K$. Order the nonzero monomials $\alpha_{p_{0}, \ldots, p_{\ell}} x_{0}^{p_{0}} x_{1}^{p_{1}} \cdots x_{\ell}^{p_{\ell}}$ of $Q$ using the lexicographic ordering on the "words" $p_{0} p_{1} \ldots p_{\ell}$. We may suppose that the coefficient of the first monomial is 1 and that $x_{0}^{m_{0}} x_{1}^{m_{1}} \cdots x_{\ell}^{m_{\ell}}$ is the monomial in question. Following the lexicographic ordering, we see that $Q$ can be written in the form

$$
Q=x_{0}^{m_{0}} \cdots x_{\ell}^{m_{\ell}}+x_{0}^{m_{0}} \cdots x_{\ell}^{1+m_{\ell}} R_{\ell}+x_{0}^{m_{0}} \cdots x_{\ell-1}^{1+m_{\ell-1}} R_{\ell-1}+\cdots+x_{0}^{m_{0}} x_{1}^{1+m_{1}} R_{1}+x_{0}^{1+m_{0}} R_{0},
$$

where $R_{j}$ belongs to $K\left[x_{k} ; k \geq j\right]$. We can then invoke Corollary 2 .

Corollary 4 If $K$ is a field, then the Krull dimension of the ring $K\left[X_{1}, \ldots, X_{\ell}\right]$ is $\ell$.

Proof. Clearly the dimension is at least $\ell$, for we have the following chain of prime ideals

$$
\{0\} \subset\left\langle X_{1}\right\rangle \subset\left\langle X_{1}, X_{2}\right\rangle \subset \cdots \subset\left\langle X_{1}, \ldots, X_{\ell}\right\rangle .
$$

To prove that the dimension of $K\left[X_{1}, \ldots, X_{\ell}\right]$ is not greater than $\ell$ it is enough in view of Corollary 3 to show that any $\ell+1$ elements in $K\left[X_{1}, \ldots, X_{\ell}\right]$ are algebraically dependent over $K$. This is a standard and elementary result (see, for instance, [4, chap. 10, Theorem 1]).

ACKNOWLEDGMENTS. Thanks to Peter Schuster, Claude Quitté, Marie-Françoise Roy, and the referee for useful comments and suggestions.

\section{References}

[1] T. Coquand T. and H. Lombardi, Hidden constructions in abstract algebra (3) Krull dimension of distributive lattices and commutative rings, in Commutative Ring Theory and Applications, M. Fontana, S.-E. Kabbaj, and S. Wiegand, eds., Lecture Notes in Pure and Applied Mathematics, no. 131, Marcel Dekker, New York, 2003, pp. $477-499$.

[2] T. Coquand, H. Lombardi, and M.-F. Roy, Une caractérisation élémentaire de la dimension de Krull (preprint, 2003).

[3] D. Eisenbud, Commutative Algebra with a View Toward Algebraic Geometry. Springer-Verlag, New York, 1995.

[4] S. Lang: Algebra, 2nd ed., Addison-Wesley, Reading, MA, 1984.

[5] H. Lombardi, Dimension de Krull, Nullstellensätze et évaluation dynamique, Math. Zeitschrift 242 (2002) $23-46$.

[6] H. Matsumura, Commutative Ring Theory, Cambridge Studies in Advanced Mathematics, no. 8, Cambridge University Press, Cambridge, 1989. 
Thierry Coquand,

Chalmers, University of Göteborg, Sweden,

email: coquand@cs.chalmers.se, http://www.cs.chalmers.se/

Henri Lombardi,

Equipe de Mathématiques, CNRS UMR 6623, UFR des Sciences et Techniques,

Université de Franche-Comté, 25030 Besançon cedex, France,

email: Henri.Lombardi@univ-fcomte.fr, http://hlombardi.free.fr/

Partially supported by the european network RAAG CT-2001-00271 